\title{
Comparative Effects of Eugenol to Bis-eugenol on Oral Mucous Membranes
}

\author{
Seiichiro FUJISAWA, Norihisa OKADA and Eitoku MURAOKA \\ Department of Oral Diagnosis, \\ School of Dentistry, Meikai University, \\ 1-1 Keyakidai, Sakado, Saitama 350-0283, Japan
}

Received April 15, 2001/Accepted June 25, 2001

\begin{abstract}
The purpose of this study was to evaluate the histopathological effect of eugenol and biseugenol on oral mucous membranes at the tissue organ level. Oral mucous membranes of mice were applied with three reagents, eugenol, bis-eugenol, and aceton (as the control). The control group showed a normal architecture. The eugenol group showed severe hyperkeratosis, parakeratosis, cellular edema, pachy chronic inflammation, pleomorphism and hyperchromatism of basal layer cells, indicating high mitotic activity. Comparatively, the biseugenol group showed mild hyperkeratosis, parakeratosis, however, the shape or arrangement of basal layer cells were normal. Bis-eugenol was considerably less toxic than eugenol.
\end{abstract}

Key words: Biocompatibility, Eugenol, Bis-eugenol

\section{INTRODUCTION}

Eugenol (4-allyl-2-methoxyphenol) is widely used not as a dental material with zinc oxide such as pulp capping cement, provisional cement, root canal sealer, and impression paste, but as a perfume ingredient. Eugenol has antioxidant, bactericidal, and sedative activities and inhibits non-enzymatic peroxidation ${ }^{1)}$. However, higher concentrations of eugenol have been reported to show some cytotoxic activity ${ }^{2)}$. In the presence of moisture the zinc oxide eugenol ( $\mathrm{ZOE}$ ) cement matrix is hydrolyzed to release eugenol ${ }^{3)}$. In general, $\mathrm{ZOE}$ cement is classified as a toxic material according to the cytotoxic standard. To improve ZOE cement, we synthesized bis-eugenol (3,3'dimethoxy-5,5'-di-2-propenyl-1,1'-biphenyl-2,2'-diol) by oxidatively producing a complex between the ortho position of the hydroxy groups in adjacent eugenol monomers, under the hypothesis that the cytotoxicity of eugenol dimer might be less than that of the original eugenol monomers, due to its lesser radicalization by the occupancy of the ortho position. The cytotoxic activity of bis-eugenol has been confirmed to be less than that of the original eugenol at the culture cell level ${ }^{4)}$ but not at the tissue organ level.

In the present study, we evaluated the histological effect of eugenol and biseugenol on oral mucous membranes. 


\section{MATERIALS AND METHODS}

Eugenol (Tokyo Kasei, Tokyo) was purifed by HPLC. The bis-eugenol used was similar to that previously reported ${ }^{4)}$. The chemical structures of eugenol and biseugenol are shown in Fig. 1. Eugenol purified by HPLC and bis-eugenol were resolved in acetone $(3 \% \mathrm{w} / \mathrm{v})$ (Tokyo Kasei, Tokyo), respectively, and the acetone was used as a control. Twelve adult mice (ICR, 8 weeks) were divided into three groups. The test reagents were applied to the central portion extended to all over the left cheek mucous membrane of the mouse using a resin carrier brush. The reagents were applied once per day for one week. On the 7th day the mouse was sacrificed, following the animal experiment guidelines, Meikai University School of Dentistry and their left cheek was excised immediately fixed in $2.5 \%$ glutaric acid for about 2 hours and then conserved in $0.1 \mathrm{M}$ sodium cacodylate. They were studied histologically using standard methods of H-E stain. Specimens were prepared routinely then observed in a transmission electron microscope (TEM : JEM-100CX).

\section{RESULTS}

The stratum corneum of the control group mice were very thin and packed closely.<smiles>C=CCc1ccc(O)c(OC)c1</smiles>

Eugenol<smiles>C=CCc1cc(C=C)c(O)c(-c2cc(C=C)cc(OC)c2O)c1</smiles>

Bis-eugenol

Fig. 1 Chemical structure of eugenol and bis-eugenol.

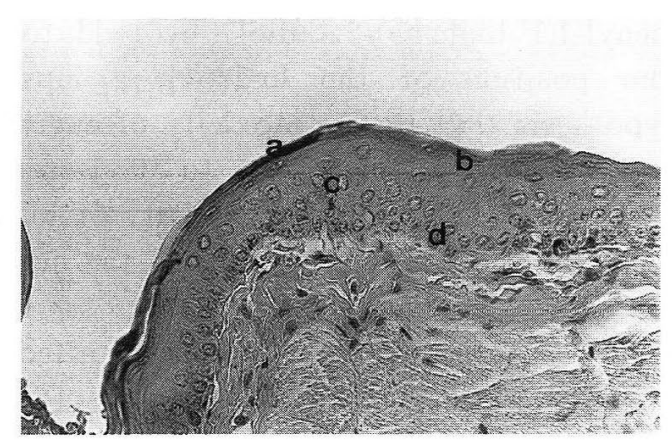

Fig. 2 Normal histological architecture of mouse labial mucosa after application of acetone. a, stratum corneum; b, granular layer; c, prickle layer; and $\mathrm{d}$, basal layer. $\times 100$. 

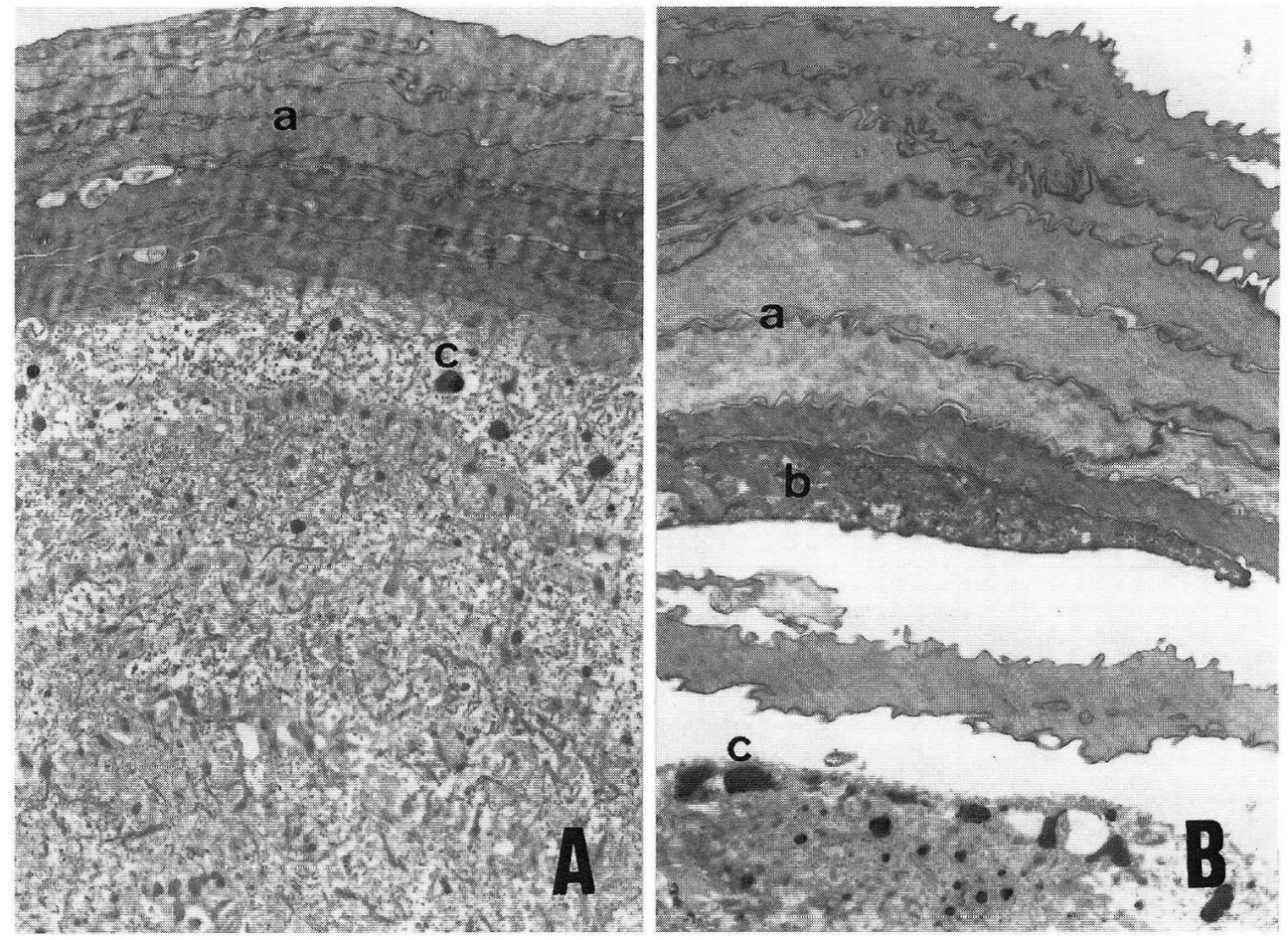

Fig. 3 A, Labial epithelium following application of acetone viewed by TEM. The stratum corneum is very thin, closely packed and about 6 cells in thickness. B, Labial epithelium following application of eugenol viewed by TEM. The stratum corneum average about 11 cells in thickness, is packed loosely and cracks disperse. In addition, shrunken nuclei remain in the layer. a, cell border line; b, shrunken nuclei; c, keratohyalin granule. $\times 3000$.

The basal layer was about one layer thickness and the cells were cuboid in shape and they were not distinguishable from the prickle layer cells. The normal histological architecture of the oral mucous membrane of mouse is shown in Fig. 2. The layer was about 6-7 cells in thickness by TEM (Figs. 3-A, 4-A). The labial epithelium following application of eugenol showed a marked thickening of the stratum corneum similar to hyperkeratosis. A prominent granular cell layer appeared typically pachy due to the chronic inflammation. Pleomorphism and hyperchromatism of the basal cell nuclei indicated high mitotic activity in this layer (Fig. 5). The stratum corneum averaged about 11-12 cells in thickness by TEM and were packed loosely, with cracks dispersed into the celluar edema. The shrunken nuclei remaining in the granular layer were indicative of parakeratosis (Fig. 3-B). The labial epithelium following application of bis-eugenol showed the shape and arrangement of nomal cells (Fig.6). The stratum corneum averaged about 8-9 cells in thickness by TEM, indicating a mild hyperkeratosis. The shrunken nuclei remaining in the granular layer were indicative of parakeratosis (Fig. 4-B). 


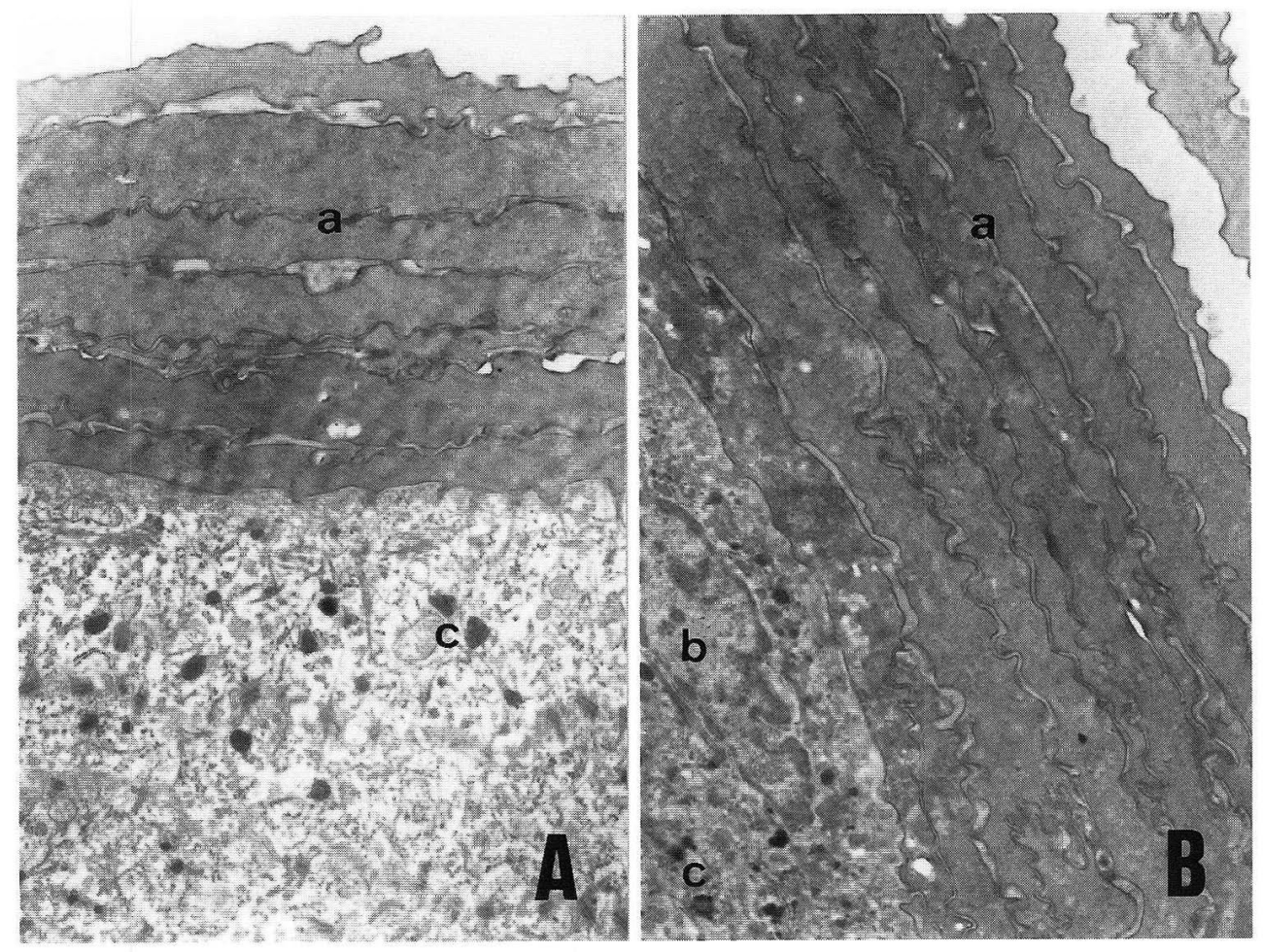

Fig. 4 A, Labial epithelium following application of acetone viewed by TEM. B, Labial epithelium following application of bis-eugenol viewed by TEM. About 8 cells in thickness and parakeratosis are seen, but the type of cells is normal. a, cell border line; b, shrunken nuclei; c, keratohyalin granule. $\times 5000$.

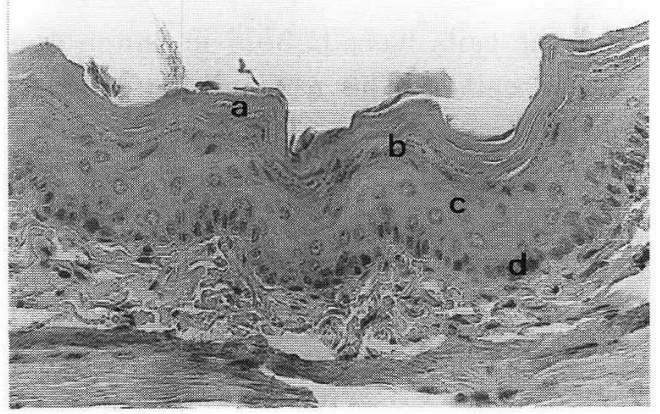

Fig. 5 Labial epithelium following application of $100 \%$ eugenol. Notice the marked thickening of the stratum corneum and prominent granular layer. a, stratum corneum; b, granular layer; c, prickle layer; and d, basal layer. $\times 100$.

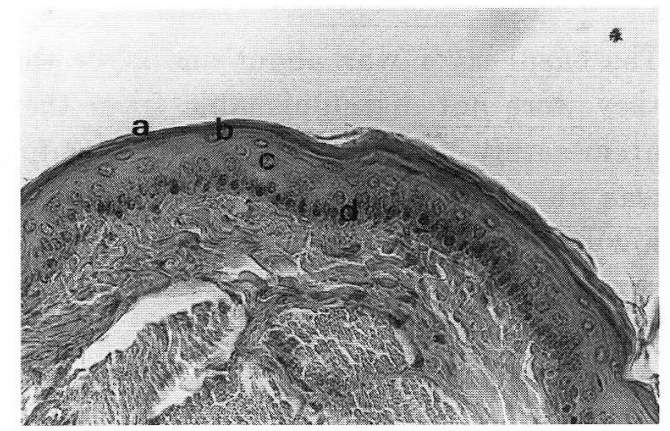

Fig. 6 Labial epithelium following application of bis-eugenol. The shape and arrangement of the cells are normal. a, stratum corneum; b, granular layer; c, prickle layer; and d, basal layer. $\times 100$. 


\section{DISCUSSION}

When we applied eugenol topically to oral mucous mouse membranes, swelling and redness could be observed in this area on the 4th day; in other words, the inflammation was confirmed grossly. However, it did not show any changes in the acetone (control) or bis-eugenol application. The degree of hyperkeratosis was arbitrarily classified into mild or severe by measuring precisely the thickness of the stratum corneum from TEM photographs. The criteria was cytoplasm surrounded by a cell line. The intercellular line was the border line of one cell layer. The findings suggested severe hyperkeratosis by eugenol application, mild hyperkeratosis by biseugenol, and normal by acetone application.

Although there was little change in the epithelium of the specimens, a marked difference was the clumping of the condensed keratohyalin in the granular layer from the eugenol applied area that was a pachy chronic inflammation. Nevertheless, we used the eugenol for producing the acute symptoms. A one day interval may be too long for the mouse model leading it into a chronic phase or recovery process. The organelles such as the shrunken nuclei left in this layer appeared typical of parakeratosis. In addition, hyperchromatism or pleomorphism of the basal layer in the eugenol group indicated high mitotic activity. Comparatively, a little dark staining of the basal layer cells and parakeratosis in the bis-eugenol group were observed, which may indicate low mitotic activity.

The direct interaction between purified eugenols and oral mucous membranes was previously reported to cause the denaturation of cystoplasmic proteins and loss of staining capacity of epithelium, loss of cell boundaries, swelling and cell necrosis, and, in addition, to exhibit vesicle formation of edema in the corium, and striated muscle dissolution ${ }^{5)}$.

We previously reported that eugenol produced radicals and radicals may be responsible for the induction of cytotoxic activity ${ }^{6-9)}$. However, eugenol is used in dentistry such as in $\mathrm{ZOE}$ cements, and the cytotoxic activity of eugenol was reported to be mild for the reaction of implants to connective tissues of Wister rats $^{10}$.

In conclusion, bis-eugenol was less toxic than eugenol at the tissue organ level.

\section{ACKNOWLEDGMENTS}

This study was supported, in part, by a Grant-in-Aid from the Ministry of Education, Science, Sports and Culture of Japan (S. Fujisawa : No.10671842).

The authors express thanks to Dr. Takeshi Oi, Department of Oral Pathology, Meikai University School of Dentistry, for his advice and support.

\section{REFERENCES}

1) Markowitz, K., Moynihan, M., Liu, M. and Kim, S.: Biologic properties of eugenol and zinc oxide-eugenol. A clinically oriented review, Oral Surg Oral Med Oral Pathol 73 : 729737, 1992. 
2) Gerosa, R., Borin, M., Menegazzi, G., Puttini, M. and Cavalleri, G.: In vitro evaluation of the cytotoxicity of pure eugenol, J Endod $22: 532-534,1996$.

3) Hume, W. R.: Influence of dentine on the pulpward release of eugenol or acids from restorative materials. J Oral Rehabil 21 : 469-473, 1994.

4) Fujisawa, S., Kashiwagi, Y., Atsumi, T., Iwakura, I., Ueha, T., Hibino, Y. and Yokoe, I.: Application of bis-eugenol to a zinc oxide eugenol cement, J Dent 27 : 291-295, 1999.

5) Kozam, G. and Mantell, G. M.: The effect of eugenol on oral mucous membranes, J Dent Res 57 : 954-957, 1978.

6) Satoh, K., Sakagami, H., Yokoe, I., Kochi, M. and Fujisawa, S.: Interaction between eugenol-related compounds and radicals, Anticancer Res $18: 425-428,1998$.

7) Satoh, K., Ida, Y., Sakagami, H., Tanaka, T. and Fujisawa, S.: Effect of antioxidants on radical intensity and cytotoxic activity of eugenol, Anticancer Res $18: 1549-1552,1998$.

8) Fujisawa, S., Atsumi, T., Satoh, K., Kadoma, Y., Ishihara, M., Okada, N., Nagasaki, M., Yokoe, I. and Sakagami, H.: Radical generation, radical-scavenging activity, and cytotoxicity of eugenol-related compounds, Vitr Mol Toxicol $13: 269-279,2000$.

9) Atsumi, T., Iwakura, I., Fujisawa, S. and Ueha, T.: Reactive oxygen species generation and photo-cytotoxicity of eugenol in solutions of various $\mathrm{pH}$, Biomaterials in press.

10) Guglani, L. M. and Allen, E. F.: Connective tissue reaction to implants of periodontal packs, J Periodontol 36 : 279-282, 1965. 